\title{
Synthetic magnetohydrodynamics in Bose-Einstein condensates and routes to vortex nucleation
}

\author{
L. B. Taylor, ${ }^{1}$ R. M. W. van Bijnen, ${ }^{2}$ D. H. J. O’Dell, ${ }^{3}$ N. G. Parker, ${ }^{4}$ S. J. J. M. F. Kokkelmans, ${ }^{2}$ and A. M. Martin ${ }^{1}$ \\ ${ }^{1}$ School of Physics, University of Melbourne, Parkville, Victoria 3010, Australia \\ ${ }^{2}$ Eindhoven University of Technology, PO Box 513, NL-5600 MB Eindhoven, The Netherlands \\ ${ }^{3}$ Department of Physics and Astronomy, McMaster University, Hamilton, Ontario, Canada L8S 4MI \\ ${ }^{4}$ School of Mathematics and Statistics, Newcastle University, Newcastle upon Tyne, United Kingdom
}

(Received 23 November 2010; published 18 August 2011)

\begin{abstract}
Engineering of synthetic magnetic flux in Bose-Einstein condensates [Lin et al., Nature (London) 462, 628 (2009)] has prospects for attaining the high vortex densities necessary to emulate the fractional quantum Hall effect. We analytically establish the hydrodynamical behavior of a condensate in a uniform synthetic magnetic field, including its density and velocity profile. Importantly, we find that the onset of vortex nucleation observed experimentally corresponds to a dynamical instability in the hydrodynamical solutions and reveal other routes to instability and anticipated vortex nucleation.
\end{abstract}

DOI: 10.1103/PhysRevA.84.021604

PACS number(s): 67.85.-d, 03.75.Kk, 47.20.-k, 73.43.Cd

One of the driving forces behind quantum degenerate gas research is the emulation of many-body condensed matter phenomenon [1]. For quantum degenerate Bose gases, achievements include the observation of Bloch oscillations [2] and the Mott insulator superfluid transition [3]. Considerable attention has also been applied to the achievement of the fractional quantum Hall $(\mathrm{FQH})$ regime in rotating BoseEinstein condensates (BECs) [4]. To obtain quantum Hall physics time reversal symmetry must be broken. In solid state devices this is done through the relatively simple process of applying a magnetic field. Since the atoms in a BEC are neutral an alternative method needs to be applied to break time reversal symmetry. In the context of FQH physics considerable focus has been applied to the breaking of time reversal symmetry through rotation.

For BECs in rotating traps the nucleation of vortices has been observed by several groups [5]. Theoretical superfluid hydrodynamical studies, in the Thomas-Fermi (TF) regime, have proved effective in calculating the rotation frequency at which vortices are nucleated by dynamical instability [6,7]. Such studies, which have been extended to dipolar BECs [8], agree with numerical predictions from the Gross-Pitaevskii equation [6,7,9-12]. However, to reach the $\mathrm{FQH}$ regime the number of vortices needs to be significantly larger than the number of bosons in the BEC. Typically, experiments are carried out in parabolic traps, defined by average in-plane trapping frequency $\omega_{\perp}$. When the rotation frequency, $\Omega_{z}$, is equal to $\omega_{\perp}$ the BEC becomes untrapped. Hence, the attainment of the FQH regime, which requires $\Omega_{z} \rightarrow \omega_{\perp}$, is an extremely challenging task. An alternative approach is to generate a synthetic vector potential [13], which breaks the time reversal symmetry of the problem, producing a synthetic magnetic field [14-20].

The recent work of Lin et al. [20] has demonstrated the effectiveness of such an approach, in a ${ }^{87} \mathrm{Rb} \mathrm{BEC}$, through the experimental realization of a synthetic vector potential in the Landau gauge: $\mathbf{A}^{*}=\mathbf{A}_{x}^{*} \hat{\mathbf{X}}$, corresponding to a synthetic magnetic field $\mathbf{B}^{*}=\nabla \times \mathbf{A}^{*}$. The authors showed that vortices were nucleated at a critical synthetic magnetic field. Here we generalize the TF methodology used to successfully calculate the onset of vortex nucleation in rotating systems $[6,7]$ to the case of synthetic magnetic fields in harmonically confined BECs. We analytically determine stationary solutions, including the aspect ratios, of a BEC and determine the critical synthetic magnetic field at which the stationary solutions become unstable. In the rotating case this instability corresponds to the onset of vortex nucleation. We find that this analysis predicts the synthetic magnetic field at which vortex nucleation occurred in the experiments of Lin et al. [20].

Our starting point is a generalized form of the GrossPitaevskii equation, which provides a mean-field description of the condensate wave function, $\psi \equiv \psi(\mathbf{r}, t)$ :

$$
i \hbar \frac{\partial \psi}{\partial t}=\left\{\frac{\left[-i \hbar \nabla-q^{*} \mathbf{A}^{*}\right]^{2}}{2 m}+V(\mathbf{r}, t)+g|\psi|^{2}\right\} \psi,
$$

where $m$ is the atomic mass, $V(\mathbf{r}, t)$ is the confining potential, and $g=4 \pi \hbar^{2} a_{s} / m$ defines the contact interactions, via the $s$-wave scattering length $a_{s}$.

Expressing $\psi=\sqrt{\rho} \exp [i \theta]$ in the TF limit we can rewrite the Gross-Pitaevskii equation as

$$
\begin{gathered}
\frac{\partial \rho}{\partial t}=-\nabla \cdot[\rho \boldsymbol{v}], \\
\frac{\partial \mathbf{v}}{\partial t}=-\nabla\left[\frac{v^{2}}{2}+\frac{1}{m}(V+g \rho)\right],
\end{gathered}
$$

where the generalized velocity is $\boldsymbol{v}=\mathbf{v}-q^{*} \mathbf{A}^{*} / m$, with $\mathbf{v}=\hbar / m \nabla \theta$.

To investigate stationary solutions we set $\partial \rho / \partial t=\partial \mathbf{v} /$ $\partial t=0$ and solve for $\rho$ and $\mathbf{v}$. Having found static solutions, they are not necessarily stable and so we analyze their dynamical stability. To do this we consider small perturbations ( $\delta \rho$ and $\delta \theta$ ) to the stationary solutions. Then, by linearizing the hydrodynamical equations, the dynamics of such perturbations are described by $[6,7]$

$$
\frac{\partial}{\partial t}\left[\begin{array}{l}
\delta \theta \\
\delta \rho
\end{array}\right]=-\left[\begin{array}{cc}
\boldsymbol{v} \cdot \boldsymbol{\nabla} & \frac{g}{\hbar} \\
\frac{\hbar}{m} \boldsymbol{\nabla} \cdot \rho_{0} \nabla & {[(\boldsymbol{\nabla} \cdot \boldsymbol{v})+\boldsymbol{v} \cdot \nabla]}
\end{array}\right]\left[\begin{array}{l}
\delta \theta \\
\delta \rho
\end{array}\right] .
$$

To investigate the stability of the BEC we look for eigenfunctions and eigenvalues of the above operator: dynamical instability arises when one or more eigenvalues possess a real positive part. The size of the real eigenvalues dictates the rate 
at which the instability grows. Imaginary eigenvalues relate to stable collective modes of the system, that is, sloshing and breathing [21]. In order to find such eigenfunctions we consider a polynomial ansatz for the perturbations in the coordinates $x$, $y$, and $z$ of total degree $n$. For the examples considered below, all operators in Eq. (4), acting on polynomials of degree $n$, result in polynomials of (at most) the same degree. Therefore, Eq. (4) can be rewritten as a scalar matrix operator, acting on vectors of polynomial coefficients, for which finding the eigenvectors and eigenvalues is a trivial task.

Below we consider the static solutions to Eqs. (2) and (3) for a BEC in a static elliptical harmonic trap under the influence of a synthetic magnetic field in the $z$ direction. The harmonic trapping potential has the form $V(\mathbf{r}, t)=m \omega_{\perp}^{2}\left[x^{2}(1-\epsilon)+\right.$ $\left.y^{2}(1+\epsilon)+z^{2} \gamma^{2}\right] / 2$. In the $x-y$ plane the trap has a mean trap frequency $\omega_{\perp}$ and ellipticity $\epsilon$. The trap strength in the axial direction is specified by $\gamma=\omega_{z} / \omega_{\perp}$. Throughout this paper we restrict our discussion to the case where $\omega_{\perp}$ and $\omega_{z}$ are greater than zero. Since the synthetic magnetic field is defined in terms of the synthetic vector potential there is more than one possible choice of $\mathbf{A}^{*}$. We consider the two most popular choices, the Landau and symmetric gauges. In each case we find the following form of the generalized velocity provides exact stationary solutions to Eqs. (2) and (3): $\boldsymbol{v}=\alpha \omega_{\perp}(y \hat{\mathbf{i}}+x \hat{\mathbf{j}})-q^{*} \mathbf{A}^{*} / m$. For the symmetric $(\mathrm{S})$ and Landau (L) gauges the synthetic vector potential has the forms: $\mathbf{A}^{*}=-\hat{\mathbf{i}} B_{z} y / 2+\hat{\mathbf{j}} B_{z} x / 2(\mathrm{~S})$ and $\mathbf{A}^{*}=-\hat{\mathbf{i}} B_{z} y(\mathrm{~L})$, such that $\mathbf{B}^{*}=\hat{\mathbf{k}} B_{z}^{*}$.

Setting $\partial \rho / \partial t=\partial \mathbf{v} / \partial t=0$ in Eqs. (2) and (3) the stationary solutions for the respective gauges are

$$
\begin{aligned}
& 0=\left(\alpha+\frac{\tilde{B}_{z}^{*}}{2}\right) \tilde{\omega}_{x}^{2}+\left(\alpha-\frac{\tilde{B}_{z}^{*}}{2}\right) \tilde{\omega}_{y}^{2} \\
& 0=\left(\alpha+\tilde{B}_{z}^{*}\right) \tilde{\omega}_{x}^{2}+\alpha \tilde{\omega}_{y}^{2}
\end{aligned}
$$

where the dimensionless effective trap frequencies are

$$
\begin{aligned}
& \tilde{\omega}_{x}^{2}=(1-\epsilon)+\alpha^{2}-\alpha \tilde{B}_{z}^{*}+\left(\frac{\tilde{B}_{z}^{*}}{2}\right)^{2} \\
& \tilde{\omega}_{x}^{2}=(1-\epsilon)+\alpha^{2} \\
& \tilde{\omega}_{y}^{2}=(1+\epsilon)+\alpha^{2}+\alpha \tilde{B}_{z}^{*}+\left(\frac{\tilde{B}_{z}^{*}}{2}\right)^{2} \\
& \tilde{\omega}_{y}^{2}=(1+\epsilon)+\alpha^{2}+2 \alpha \tilde{B}_{z}^{*}+\left(\tilde{B}_{z}^{*}\right)^{2}
\end{aligned}
$$

and $\tilde{B}_{z}^{*}=q^{*} B_{z}^{*} / m \omega_{\perp}$. Equations (5), (7), and (9) [Eqs. (6), (8), and (10)] can be solved analytically to determine $\alpha$ in the symmetric [Landau] gauge as a function of $\epsilon$ and $\tilde{B}_{z}^{*}$, with the solutions being independent of the axial trapping strength $\gamma[6,7,23]$.

In Fig. 1(a) we plot the solutions $\alpha$ for the symmetric and Landau gauges, respectively, for $\epsilon=0$. In each case for $\tilde{B}_{z}^{*}<2$ only one solution exists with two additional solutions, referred to as the upper and lower branch solutions, bifurcating at $\tilde{B}_{z}^{*}=2$. Despite the quantitative differences in the solutions for $\alpha$ the generalized velocities, relevant in determining the physical properties of the BEC, are gauge invariant.

The solutions for $\alpha$ [Fig. 1(a)] have qualitatively similar properties to the solutions obtained in the TF limit for a BEC in a rotating trap. This similarity is best observed through a
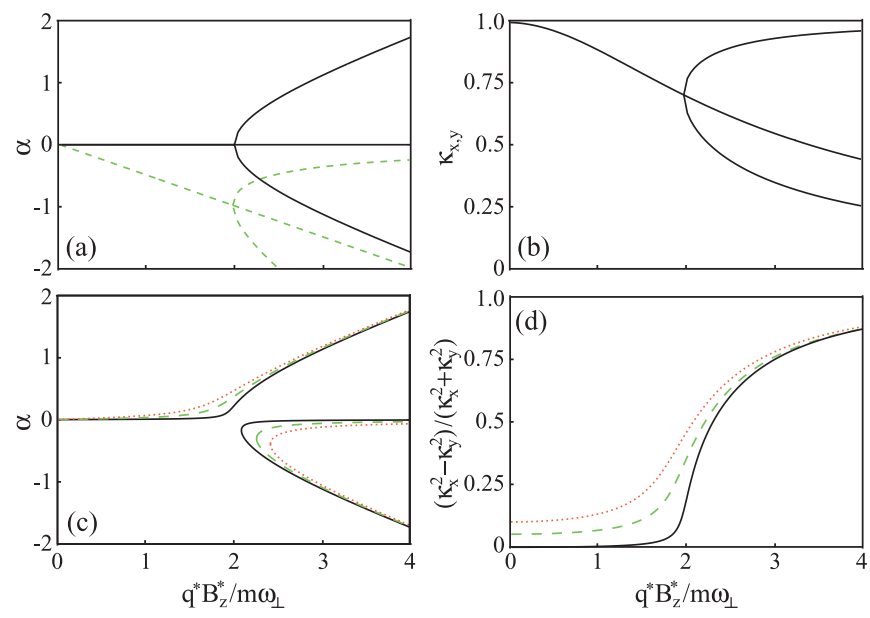

FIG. 1. (Color online) (a) For a spherically symmetric trap $\alpha$ as a function of the synthetic magnetic field strength in the symmetric (solid black curve) and Landau (dashed green curve) gauges. (b) $\kappa_{x, y}$ as a function of $\tilde{B}_{z}^{*}$ in the symmetric and Landau gauges [22]. (c) $\alpha$ as a function of the synthetic magnetic field (symmetric gauge). (d) The condensate ellipticity $\left(\kappa_{x}^{2}-\kappa_{y}^{2}\right) /\left(\kappa_{x}^{2}+\kappa_{y}^{2}\right)$ for the upper branch solutions in (c) as a function of the synthetic magnetic field strength [22]. In (c) and (d) $\epsilon=0.01$ (solid black curve), $\epsilon=0.05$ (dashed green curve), and $\epsilon=0.1$ (red dotted curve).

comparison of the symmetric gauge solutions with solutions for a BEC in a rotating trap $[6,7,23]$. In a rotating trap $\alpha$ is determined by Eq. (5), with $\tilde{B}_{z}^{*} / 2$ replaced by the dimensionless rotation frequency about the $z$ axis $\left(\tilde{\Omega}_{z}=\Omega_{z} / \omega_{\perp}\right)$, with terms proportional to $\left(\tilde{B}_{z}^{*}\right)^{2}$ in Eqs. (7) and (9) set to zero. This difference emphasizes the key advantage a synthetic magnetic field has over physical rotation. For physical rotation, when $\tilde{\Omega}_{z} \rightarrow 1, \tilde{\omega}_{x}\left(\tilde{\omega}_{y}\right)$ tends to zero for the upper (lower) branch solution, hence the BEC is no longer confined. In the synthetic magnetic field case, the term proportional to $\left(\tilde{B}_{z}^{*}\right)^{2}$ prevents $\tilde{\omega}_{x, y}$ tending to zero and the BEC remains confined.

We now consider how the synthetic magnetic field alters the TF condensate density profile:

$$
\rho=\rho_{0}\left(1-\frac{x^{2}}{R_{x}^{2}}-\frac{y^{2}}{R_{y}^{2}}-\frac{z^{2}}{R_{z}^{2}}\right),
$$

with $\rho_{0}=15 N /\left(8 \pi R_{x} R_{y} R_{z}\right)$. In Fig. 1(b) we plot the aspect ratios of the condensate $\kappa_{x, y}^{2}=R_{x, y}^{2} / R_{z}^{2}=\gamma^{2} / \tilde{\omega}_{x, y}^{2} \quad\left[R_{z}^{2}=\right.$ $\left.2 g \rho_{0} /\left(m \omega_{z}^{2}\right)\right]$ as a function of the synthetic magnetic field, for both the symmetric and Landau gauges, with $\gamma=1$ and $\epsilon=0$. The solutions for $\kappa_{x, y}$ are gauge independent, with the solutions bifurcating at $\tilde{B}_{z}^{*}=2$. For low synthetic magnetic fields, $\tilde{B}_{z}^{*}<2$, the aspect ratios of the condensate in the $x$ and $y$ directions are reduced by $>25 \%$, with $\kappa_{x}=\kappa_{y}$. For $\tilde{B}_{z}^{*}>2$, consistent with the solutions obtained for $\alpha$ we have three solutions for $\kappa_{x, y}$. Since the solutions are gauge independent, with no loss of generality, we describe the dependence of the aspect ratios, for $\tilde{B}_{z}^{*}>2$, in terms of the solutions for $\alpha$ in the symmetric gauge [Fig. 1(a), solid black curve]. For the $\alpha=0$ solution the aspect ratios of the condensate continue to reduce with $\kappa_{x}=\kappa_{y}$. For the upper (lower) branch solutions for $\alpha \kappa_{x}$ follows the upper (lower) branch solutions and $\kappa_{y}$ 

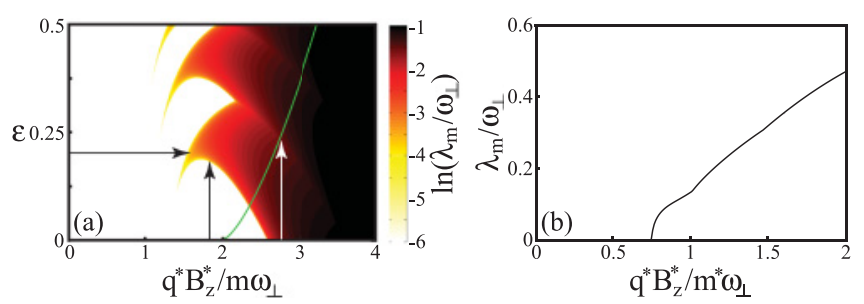

FIG. 2. (Color online) (a) The logarithm of the maximum positive real eigenvalues of Eq. (4) $(n=5)$ for the upper branch solutions of $\alpha$ as a function of $\tilde{B}_{z}^{*}$ and $\epsilon$. The solid green curve plots the trap ellipticity $(\epsilon)$ at which the backbending point $\left(\tilde{B}_{z(\mathrm{~b})}^{*}\right)$ occurs [22] and the horizontal and vertical arrows indicate routes to instability. (b) For the experimental parameters of Lin et al. [20,25], for the $\alpha$ branch which starts at $\alpha=0$ for $\tilde{B}_{z}^{*}=0$, the maximum positive real eigenvalues of Eq. (4) $(n=5)$.

follows the lower (upper) branch solutions in Fig. 1(b), with $\kappa_{x}(\alpha)=\kappa_{y}(-\alpha)$.

For $\epsilon \neq 0$ the solutions for $\alpha$ significantly change [Fig. 1(c)]. For the symmetric gauge, the $\alpha=0$ solution no longer exists and the plot has two distinct branches. The upper branch $(\alpha \geqslant 0)$ is single valued and exists over all $\tilde{B}_{z}^{*}$. For this branch the ellipticity of the BEC in the $x-y$ plane is plotted as a function of the synthetic magnetic field, in Fig. 1(d). We find that the BEC ellipticity monotonically increases from $\epsilon$ with increasing $\tilde{B}_{z}^{*}$. The lower branch $(\alpha<0)$ is double valued and exists only when $\tilde{B}_{z}^{*}$ is greater than the backbending magnetic field: $\tilde{B}_{z(\mathrm{~b})}^{*}(\epsilon)$. This lower branch backbending point shifts to higher $\tilde{B}_{z}^{*}$ as the ellipticity is increased [solid green in Fig. 2(a)] [22]. In Fig. 2(a) we have characterized the stability of the upper branch solutions through the evaluation of Eq. (4) for polynomial perturbations up to order $n=5$ [22]. The instability region consists of a series of crescents [6]. Each crescent corresponds to a single value of the polynomial degree $n$, where higher values of $n$ add extra crescents, from above. At the high synthetic field end these crescents merge to form a main region of instability, characterized by large eigenvalues. At low synthetic magnetic fields the crescents become vanishingly small, with eigenvalues several orders of magnitude smaller than in the main instability region. These regions induce instability if they are traversed very slowly [24]. $\tilde{B}_{z(\mathrm{~b})}^{*}(\epsilon)$ and the stability of the stationary solutions of the upper branch are key to understanding the response of the BEC to the adiabatic introduction of $\epsilon$ or $\tilde{B}_{z}^{*}$.

For a fixed synthetic magnetic field as the ellipticity of the trap is introduced adiabatically, from zero, the BEC can follow two routes, depending on the value of $\tilde{B}_{z}^{*}$ relative to $\tilde{B}_{z(\mathrm{~b})}^{*}(0)$. For $\tilde{B}_{z}^{*}<\tilde{B}_{z(\mathrm{~b})}^{*}(0)$ the solutions follow the upper branch until these solutions become dynamically unstable. This route to instability is schematically indicated by the vertical black arrow in Fig. 2(a). For $\tilde{B}_{z}^{*}>\tilde{B}_{z \text { (bif) }}^{*}(0)$ the BEC follows the lower branch from $\alpha=0$ to negative $\alpha$. However, as $\epsilon$ is increased $\tilde{B}_{z(\mathrm{~b})}^{*}(\epsilon)$ shifts upward [solid green curve in Fig. 2(a)]. When $\tilde{B}_{z}^{*}>\tilde{B}_{z(\mathrm{~b})}^{*}(\epsilon)$ the lower branch no longer exists. This route to instability is schematically indicated by the vertical white arrow in Fig. 2(a). For fixed ellipticity as the synthetic magnetic field is introduced adiabatically, from zero, the BEC follows the upper branch solutions for $\alpha$, which become dynamically unstable. This route to instability is schematically indicated by the horizontal black arrow in Fig. 2(a).

In the case of rotation [7] the analogous instabilities due to (i) the dynamical instability of the upper branch solutions or (ii) the shifting of the backbending point lead to the nucleation of vortices. As such we expect that for (i) the adiabatic introduction of a synthetic magnetic field for a fixed trap ellipticity or (ii) the adiabatic introduction of trap ellipticity for a fixed synthetic magnetic field, the onset of vortex nucleation can be determined by the onset of instability in the upper branch solutions and the evolution of the backbending point.

To test this we consider the recent experiment of Lin et al. [20] where the synthetic magnetic field was generated via an engineered, Landau gauge, vector potential [18]. To engineer the vector potential an ${ }^{87} \mathrm{Rb} \mathrm{BEC}$ was illuminated with a pair of Raman laser beams with a momentum difference along the $x$ direction, producing three tunable dressed states. The dressed state with the lowest energy corresponds to the following Hamiltonian along the $x$ direction $H_{x}=\left(\hbar k_{x}-q^{*} A_{x}^{*}\right)^{2} / 2 m^{*}$. In this Hamiltonian $A_{x}^{*}$ is the synthetic vector potential, controlled by the Zeeman shift for the atoms with synthetic charge $q^{*}$ and an effective mass for the atoms along the $x$ direction $m^{*}$. To produce the desired spatially synthetic vector potential the Zeeman shift varies linearly in the $y$ direction, achieved by the application of a magnetic field gradient along $y$, resulting in an effective vector potential $A_{x}^{*}=-B_{z}^{*} y$. Including the effective mass into the hydrodynamical analysis:

$$
\begin{aligned}
\boldsymbol{v} & =\left(\frac{\hbar}{m^{*}} \frac{\partial \theta}{\partial x}+\frac{q^{*} B_{z}^{*} y}{m^{*}}\right) \hat{\mathbf{i}}+\frac{\hbar}{m} \frac{\partial \theta}{\partial y} \hat{\mathbf{j}} \\
& =\left(\frac{m}{m^{*}} \alpha \omega_{\perp} y+\frac{q^{*} B_{z}^{*} y}{m^{*}}\right) \hat{\mathbf{i}}+\alpha \omega_{\perp} x \hat{\mathbf{j}},
\end{aligned}
$$

the stationary solutions are determined by

$$
0=\left(\frac{m}{m^{*}} \alpha+\tilde{B}_{z}^{*}\right) \tilde{\omega}_{x}^{2}+\alpha \tilde{\omega}_{y}^{2}
$$

with $\tilde{B}_{z}^{*}=q^{*} B_{z}^{*} /\left(m^{*} \omega_{\perp}\right), \tilde{\omega}_{x}^{2}$ defined by Eq. (8) and

$$
\tilde{\omega}_{y}^{2}=(1+\epsilon)+\frac{m}{m^{*}} \alpha^{2}+2 \alpha \tilde{B}_{z}^{*}+\frac{m^{*}}{m}\left(\tilde{B}_{z}^{*}\right)^{2} .
$$

In the experiments the synthetic magnetic field is ramped up over $0.3 \mathrm{~s}$. Thus as the synthetic field is increased the solution starting at $\alpha=0$ when $\tilde{B}_{z}^{*}=0$ will be tracked, until it becomes dynamically unstable. Experimentally vortex nucleation started when the synthetic magnetic flux $\left(\Phi_{B_{z}^{*}}=\right.$ $\left.\pi R_{x} R_{y} B_{z}^{*}\right)$, in units of the flux quantum $\left(\Phi_{0}=h / q^{*}\right)$, passing through the BEC

$$
\frac{\Phi_{B_{z}^{*}}}{\Phi_{0}}=\frac{\tilde{B}_{z}^{*}}{2}\left(15 N \tilde{a}_{s} \gamma\right)^{\frac{2}{5}}\left(\tilde{\omega}_{x} \tilde{\omega}_{y}\right)^{-\frac{3}{5}} \approx 10,
$$

see Fig. 2(g) of Ref. [20]. In Eq. (15) we have redefined the contact interactions to include the effective mass difference in the $x$ direction, such that $\tilde{a}_{s}=a_{s} /\left(2.5^{1 / 3} l_{x y}\right)$, with $l_{x y}=\sqrt{\hbar / m \omega_{\perp}}$. Using the experimental parameters [25] the stationary solution becomes unstable when $\tilde{B}_{z}^{*}=0.74$ [Fig. 2(b)], corresponding to $\frac{\Phi_{B}}{\Phi_{0}}=11.1$, in close agreement with the experimental observation of the onset of vortex nucleation. This strongly suggests that the observed onset of vortex nucleation arises 
from a dynamical instability as calculated from the stationary TF solutions [26].

The engineering of synthetic magnetic fields, in BECs, offers a new and exciting route into the emulation of $\mathrm{FQH}$ physics. Such an arrangement also provides a new route to investigate the $\mathrm{FQH}$ regime in lattice systems, without the need to rotate the lattice. By adapting the techniques developed by Sinha and Castin [6] we have shown that the nucleation of vortices, the first step in obtaining high density vortex systems, due to adiabatic changes in the synthetic field and ellipticity of the trap can be calculated in the TF regime. This enables us to analytically determine the aspect ratios of the BEC for fields below the nucleation point and show that the experimental observation of the onset of vortex nucleation is due to dynamical instabilities in the stationary state of the BEC.

We acknowledge financial support from the Natural Sciences and Engineering Research Council of Canada (DHJOD) and the TU/e Fund for Excellence (RMWvB and SJJMFK).
[1] I. Bloch, J. Dalibard, and W. Zwerger, Rev. Mod. Phys. 80, 885 (2008).

[2] M. Fattori et al., Phys. Rev. Lett. 100, 080405 (2008).

[3] D. Jaksch, C. Bruder, J. I. Cirac, C. W. Gardiner, and P. Zoller, Phys. Rev. Lett. 81, 3108 (1998); M. Greiner et al., Nature (London) 415, 39 (2002); M. Köhl et al., J. Low Temp. Phys. 138, 635 (2005); I. B. Spielman, W. D. Phillips, and J. V. Porto, Phys. Rev. Lett. 98, 080404 (2007); 100, 120402 (2008); J. Mun et al., ibid. 99, 150604 (2007).

[4] N. R. Cooper and N. K. Wilkin, Phys. Rev. B 60, 16279(R) (1999); N. K. Wilkin and J. M. F. Gunn, Phys. Rev. Lett. 84, 6 (2000); N. R. Cooper, N. K. Wilkin, and J. M. F. Gunn, ibid. 87, 120405 (2001); U. R. Fischer and G. Baym, ibid. 90, 0140402 (2003); N. Regnault and Th. Jolicoeur, ibid. 91, 030402 (2003); V. Bretin, S. Stock, Y. Seurin, and J. Dalibard, ibid. 92, 050403 (2004); V. Schweikhard, I. Coddington, P. Engels, V. P. Mogendorff, and E. A. Cornell, ibid. 92, 040404 (2004); N. Regnault and Th. Jolicoeur, Mod. Phys. Lett. B 18, 1003 (2004); M. A. Baranov, K. Osterloh, and M. Lewenstein, Phys. Rev. Lett. 94, 070404 (2005).

[5] K. W. Madison, F. Chevy, W. Wohlleben, and J. Dalibard, Phys. Rev. Lett. 84, 806 (2000); K. W. Madison, F. Chevy, V. Bretin, and J. Dalibard, ibid. 86, 4443 (2001); E. Hodby, G. Hechenblaikner, S. A. Hopkins, O. M. Marago, and C. J. Foot, ibid. 88, 010405 (2001).

[6] S. Sinha and Y. Castin, Phys. Rev. Lett. 87, 190402 (2001).

[7] N. G. Parker, R. M. W. van Bijnen, and A. M. Martin, Phys. Rev. A 73, 061603(R) (2006).

[8] R. M. W. van Bijnen, D. H. J. O’Dell, N. G. Parker, and A. M. Martin, Phys. Rev. Lett. 98, 150401 (2007); A. M. Martin et al., Las. Phys. 18, 322 (2008); R. M. W. van Bijnen, A. J. Dow, D. H. J. O’Dell, N. G. Parker, and A. M. Martin, Phys. Rev. A 80, 033617 (2009).
[9] C. Lobo, A. Sinatra, and Y. Castin, Phys. Rev. Lett. 92, 020403 (2004).

[10] N. G. Parker and C. S. Adams, Phys. Rev. Lett. 95, 145301 (2005).

[11] N. G. Parker and C. S. Adams, J. Phys. B 39, 43 (2006).

[12] T. M. Wright, R. J. Ballagh, A. S. Bradley, P. B. Blakie, and C. W. Gardiner, Phys. Rev. A 78, 063601 (2008).

[13] R. Dum and M. Olshanii, Phys. Rev. Lett. 76, 1788 (1996).

[14] J. Dalibard et al., Accepted for Rev. Mod. Phys., e-print arXiv:1008.5378v1.

[15] J. Ruostekoski, G. V. Dunne, and J. Javanainen, Phys. Rev. Lett. 88, 180401 (2002).

[16] D. Jaksch and P. Zoller, New J. Phys. 5, 56 (2003).

[17] E. J. Mueller, Phys. Rev. A 70, 041603(R) (2004).

[18] I. B. Spielman, Phys. Rev. A 79, 063613 (2009).

[19] D. R. Murray, P. Ohberg, D. Gomila, and S. M. Barnett, Phys. Rev. A 79, 063618 (2009).

[20] Y.-J. Lin et al., Nature (London) 462, 628 (2009).

[21] R. M. W. van Bijnen, N. G. Parker, S. J. J. M. F. Kokkelmans, A. M. Martin, and D. H. J. O'Dell, Phys. Rev. A 82, 033612 (2010).

[22] This result is independent of the chosen gauge.

[23] A. Recati, F. Zambelli, and S. Stringari, Phys. Rev. Lett. 86, 377 (2001).

[24] I. Corro, N. G. Parker, and A. M. Martin, J. Phys. B 40, 3615 (2007).

[25] $N=1.4 \times 10^{5}, a_{s}=5 \times 10^{-9} \mathrm{~m}, m^{*}=2.5 \mathrm{~m}, \omega_{x}=80 \pi \mathrm{rad} / \mathrm{s}$, $\omega_{y}=60 \pi \mathrm{rad} / \mathrm{s}\left(\omega_{\perp}=320 \mathrm{rad} / \mathrm{s}\right.$ and $\left.\epsilon=0.38\right)$ and $\gamma=1.37$ [20]. These parameters correspond to an experimental detuning gradient $\delta^{\prime}$ of $0.2 \mathrm{kHz} \mu \mathrm{m}^{-1}$. For precise details of the dependence of the effective mass and trap parameters on the detuning gradient see Refs. [18,20].

[26] Experimental parameters, $N \tilde{a}_{s}=350$, imply the system is in the TF regime. 